\title{
Noncovalent Interaction between Gold Nanoparticles and Multiwalled Carbon Nanotubes via an Intermediatory
}

\author{
Jui-Ming Yeh, ${ }^{1}$ Kuan-Yeh Huang, ${ }^{1}$ Su-Yin Lin, ${ }^{1}$ Yu-Yao Wu, ${ }^{1}$ Chao-Chen Huang, 1 \\ and Shir-Joe Liou ${ }^{2}$ \\ ${ }^{1}$ Department of Chemistry and Center for Nanotechnology (CYCU) and R\&D Center for Membrane Technology, \\ Chung Yuan Christian University, Chung Li 32023, Taiwan \\ ${ }^{2}$ Material and Chemical Research Laboratories (ITRI), Industrial Technology Research Institute, HsinChu 310, Taiwan
}

Correspondence should be addressed to Jui-Ming Yeh, juiming@cycu.edu.tw

Received 9 September 2008; Revised 5 January 2009; Accepted 3 February 2009

Recommended by Valery N. Khabashesku

A new and effective method has been developed where self-assembled gold nanoparticles (Au-NPs) of $\sim 10 \mathrm{~nm}$ diameter are successfully attached onto the surface of sidewalls and ends of thiol-terminated multi-walled carbon nanotubes (MWNTs) functionalized with orthomercaptoaniline, acting as a bridging agent. It can bridge the carbon nanotubes (CNTs) and Au-NPs via the bi-functional moiety with benzene unit at one end and thiol group at the other end by self-assembly. The ortho-mercaptoaniline was first grafted onto the surface of the CNTs via $\pi-\pi$ interaction between the benzene ring of the mercaptoaniline and $\pi$ conjugated body of MWNTs surface to produce thiol-terminated CNTs. The bare surface of Au-NPs facilitates to attach on the thiol group of the thiol-terminated CNTs. Attenuated total reflectance FTIR, UV-visible, Raman spectroscopy and X-ray powder diffraction studies were used to verify whether the mercapto-benzene moieties have been attached to the $\pi$-conjugated body of functionalized MWNTs. The direct evidence is obtained from transmission electron microscope (TEM) images where selfassembled Au-NPs are attached onto the functionalized MWNTs.

Copyright () 2009 Jui-Ming Yeh et al. This is an open access article distributed under the Creative Commons Attribution License, which permits unrestricted use, distribution, and reproduction in any medium, provided the original work is properly cited.

\section{Introduction}

Recently, nanotechnology has become an important field in the science and technology, allowing one to manipulate matter at the nanometer scale and to incorporate nanostructures and nano-processes into working technological innovations [1-5]. Considerable interest has been focused on the possibility of construction of new assemblies from nanoparticles (NPs) and other components to yield superstructure nanomaterials with unique and useful optical, electronic, and magnetic properties [1-4].

Carbon nanotubes (CNTs), discovered by Iijima and Ichihashi [6], have attracted significant interest for the application in fabricating a new class of advanced materials like nanoelectronic devices due to their unique structural, mechanical, and electronic properties [7-12]. CNTs are potentially excellent one-dimensional nanoscale materials because of their excellent physical properties [9] and morphology that can be carefully functionalized $[10,11]$.
Nanoparticles (NPs), such as metal and semiconductor colloids with diameters of one- to ten-nanometers, are particularly attractive candidates [13-19]. Metal and semiconducting NPs have already been used as the functional components of nanoscale electronic devices [13, 14]. Au-NPs are one of the noble metal nanostructures that possess many outstanding material properties such as special optical properties, unusual electronic properties including conductivity by activated electron hopping, and remarkably high catalytic activity [20,21].

Recently, both single-walled carbon nanotubes (SWNTs) and MWNTs have been proposed as substrates for biological devices $[7,8]$. Examples of nanotube-based devices include single-electron transistors [9, 22], molecular diodes [23], memory elements [24], and logic gates [25, 26]. Metalfilled CNTs [27] also have promising applications in heterogeneous catalysis [28]. There are considerable interests in devising strategies to individually address each molecular unit, and interconnect them, without adversely affecting the 
local electronic structure [25]. Chen et al. [29] reported that small molecule with rich conjugated $\pi$ electrons such as pyrene derivatives could be bound onto the surface of carbon nanotubes through noncovalent $\pi-\pi$ interactions. Moreover, there are some reports where functional groups could be bounded directly on the surface of carbon nanotubes through covalent or noncovalent methods [13-19, 29-36]. Subsequently, Zanella et al. [37] developed a simple direct solvent-free procedure to anchor the gold nanoparticles on the surface of MWNTs functionalized with aliphatic dithiols and aminothiols. Small gold particles with narrow particle size distribution were obtained for MWNTs derivatized with 1, 6-hexanedithiol. This approach could be efficient to attach other nanostructures to CNTs through the dithiol interlinkers.

Recently, Pan et al. [38] reported where the Au-NPs are attached on MWNT through the formation of imide linkage $-(\mathrm{C}=\mathrm{O}) \mathrm{NH}$ - in Au-MWNT conjugates by several steps and drastic reaction condition. Therefore, it can be realized that different CNTs could also be interconnected through chemical functionalization [39] that may be by covalent or noncovalent interaction. The covalent modifications work well in some cases for dispersing CNTs in organic solvents, but to be useful for dispersing CNTs in polymers the extention the surface has to be modified significantly alter the mechanical and electrical properties of the CNTs. Nevertheless, noncovalent methods based on the adsorbed molecule with CNTs sidewalls through van der Waals and other interactions are preferable than that of covalent methods because they allow the excellent electronic, mechanical properties, and also preserve their unique properties of CNTs to be retained [40, 41].

In this study, we develop a new approach that comprises fewer synthetic steps and a relatively mild reaction condition. Our main purpose is to anchor the Au-NPs of $\sim 10 \mathrm{~nm}$ diameter to the sidewalls and ends of CNTs through $\pi-\pi$ interaction using orthomercaptoaniline as an interlinker. Being a bi-functionalized molecule, orthomercaptoaniline can be bound to the surface of CNTs through $\pi-\pi$ interaction between its mercaptobenzene unit in one end and the sidewall of CNTs to preserve the $\mathrm{sp}^{2}$ nanotube structure and their electronic characteristics. In other end of orthomercaptoaniline, the terminated thiol group can be attached to the surface of Au-NPs. Thus Au-NPs can be bound to the surface of CNTs through orthomercaptoaniline by taking the advantage of strong thiol-Au interaction, just as shown in Scheme 1. This assembly was confirmed by TEM, FTIRATR, UV-visible, Raman spectroscopy, and X-ray powder diffraction studies.

\section{Experimental Details}

2.1. Chemicals and Instrumentation. Multiwalled carbon nanotubes (MWNT, 95\%) and orthomercaptoaniline (95\%) were purchased from TCI (Japan) and Desunnano Co., Ltd. (Taiwan), respectively. Trisodium citrate dehydrate $\left(\mathrm{C}_{6} \mathrm{H}_{5} \mathrm{O}_{7} \mathrm{Na}_{3}\right)(100 \%$, J.T Baker) was employed as a reducing agent. Tetrachloroauric (III) acid $\left(\mathrm{HAuCl}_{4}\right)$ (99.99\%, Alfa Aesar) is used for the preparation of Au nanoparticles.

The morphology of the resulting precipitates was characterized by high-resolution transmission electron microscopy (HRTEM) and measured in a JEOL-1200 EXII TEM operated at $120 \mathrm{KV}$. Transmission-mode Fourier transform infrared (FTIR) spectroscopy was carried out in JASCO model 460 Plus spectrophotometer. The binding of colloidal gold to MWNTs was also monitored by UV-vis spectroscopy on a Cary spectrometer operated at a resolution of $1 \mathrm{~nm}$. XRD patterns were taken by PANalytical X'Pert Pro diffractometer in the range of $10-70^{\circ} 2 \theta$ range. Raman spectra were recorded with a Jobin Yvon Triax 320 using an argon ion laser operating at $514.5 \mathrm{~nm}$ with a CCD detector. The final spectrum presented is an average of three spectra recorded at different regions over the entire sample range.

\subsection{Preparation}

2.2.1. Preparation of Gold Nanoparticle. In a typical procedure, A $100 \mathrm{~mL}$ of $1 \mathrm{mM}$ aqueous $\mathrm{HAuCl}_{4}$ was reduced by $20 \mathrm{~mL}$ of $1 \mathrm{mM} \mathrm{C}_{6} \mathrm{H}_{5} \mathrm{O}_{7} \mathrm{Na}_{3}$ at $100^{\circ} \mathrm{C}$, when the color changes from pale yellow to fresh red, the solution was removed from the hot plate at that moment and cooled down at room temperature. A colloidal solution containing AuNPs of $\sim 10 \mathrm{~nm}$ diameter to sidewalls was thus obtained.

\subsubsection{Preparation of Thiol-Functionalized MWNT. Thiol-} functionalized MWNTs (MWNT-SH) were prepared by the following successful model reaction. The raw MWNT (100 mg) suspended in $5 \mathrm{~mL}$ of orthomercaptoanilines was stirred magnetically and refluxed at $150^{\circ} \mathrm{C}$ in the dark place for 12 hours the as-obtained solution gradually becomes brownish and then turns to dark. The mixture was subsequently filtered by MFS membrane filters (pore size ca. $0.45 \mu \mathrm{m}$ ), and the as-obtained filtered solid was further dried under dynamic vacuum oven for 24 hours at $60^{\circ} \mathrm{C}$.

2.2.3. Attachment of Gold Nanoparticles onto MWNTs. The MWNT-SH $(10 \mathrm{mg})$ was added to the $20 \mathrm{~mL}$ ethanol under vigorous stirring condition and then the $20 \mathrm{~mL}$ of $1 \mathrm{mM}$ colloidal Au solution was subsequently added to the MWNTs/ethanol solution. After one day, the product (i.e., Au-MWNT-SH complex) was filtered and washed several times with distilled water and finally dried in vacuum over a night.

\section{Results and Discussion}

3.1. Au-MWNT-SH Complex. The experimental procedures for samples preparation are illustrated in Scheme 1. It has been shown that the $\pi-\pi$ stacking [15] is the strong interactions between the basal plane of CNTs and the aromatic benzene ring of orthomercaptoanilines, which was indicated by the increased amount of orthomercaptoaniline linked to MWNTs. The Au-NPs are attached to the thiol group of the functionalized MWNTs due to surface-active property of the Au-NPs and strong thiol-Au interaction $[15,16]$. At 


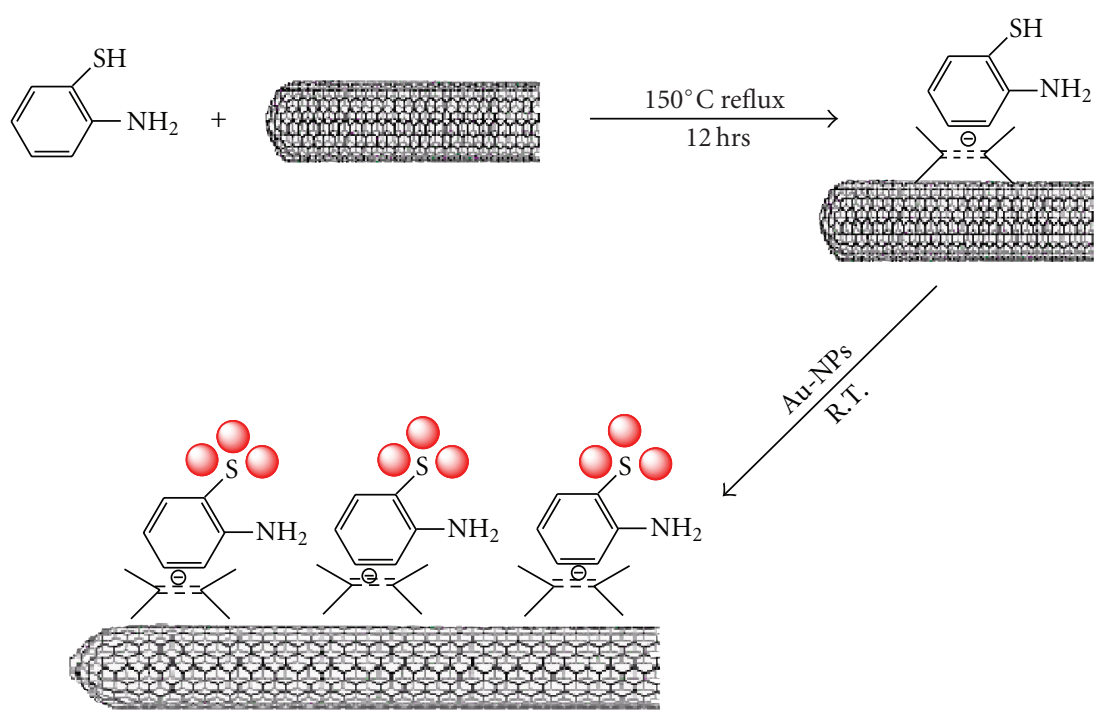

Scheme 1: Schematic illustration of thiol-terminated MWNTs, and binding of Au-NPs onto the surface of MWNT-SH as a non-covalaent approch.

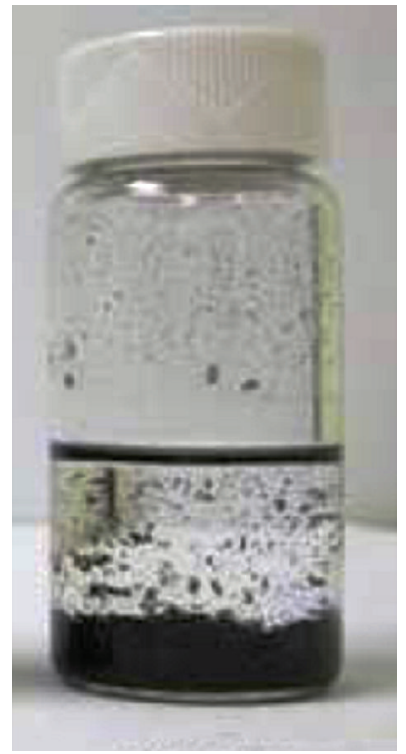

(a)

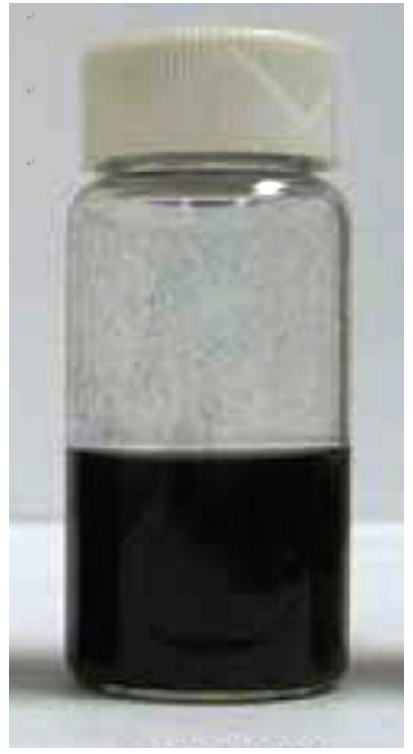

(b)
FIGURE 1: The simple dispersion test of MWNT (a) and MWNT-SH (b).

first orthomercaptoaniline formed the complex with carbon nanotubes via strong $\pi-\pi$ stacking around the whole surface of CNTs. As a simple observation, the orthomercaptoaniline functionalized CNTs exhibited a better dispersion capability in ethanol than that of raw CNTs, as shown in Figure 1. The newly formed complex abbreviated as MWNT-SH, is then reacts with Au-nanoparticle. The gold NPs forms AuMWNT-SH complex due to its strong affinity towards the sulfur. This complex formation is confirmed by the following studies.
3.2. TEM Characterization. The successful self assembly of Au-NPs to MWNT surface was confirmed by TEM studies. A micrograph of $10 \mathrm{~nm}$ Au-NPs and micrograph of a MWNT$\mathrm{SH}$ are shown in Figures 2(a) and 2(b), respectively. We observed that the assembly of Au-NPs is attached onto the surface of MWNT-SH, as shown in Figure 2(c). The dispersion for Au-NPs is large and the deposition is not quite uniform. The Au-NPs are attached through a well-defined scheme that involves the interaction of the sites on the nanotube walls decorated by thiol functional groups, coming from orthomercaptoaniline. Bare surface was formed during the preparation of Au-NPs. The Au-NPs were attached to the thiol groups of the functionalized MWNTs due to the advantage of their bare surface. After formation of this assembly, the Au-NPs act as cores to develop, which leads to some aggregation between the adjacent particles. This problem can be avoided if we employ appreciated method during the preparation of Au-NPs, sometimes bare surface Au-NPs have lots of advantages in the practical applied field. Our decoration technique presents an excellent method for monitoring the presence of these chemical groups on the surfaces of nanotubes. When the Au-NPs were mixed with MWNTs under the same condition, only few Au-NPs can be found on the surface of MWNTs. It was also noted that the interaction between the gold nanoparticles and nanotubes is quite strong, because washing and centrifugation do not affect them. It is also worth mentioning that the Au-MWNT$\mathrm{SH}$ assembly thus formed is very stable.

3.3. FTIR Spectra. Figure 3 shows the ATR-FTIR spectra of the MWNTs, orthomercaptoaniline, MWNT-SH, and AuMWNT-SH complex in the absorbance mode. The peak near $1537 \mathrm{~cm}^{-1}$ corresponds to the IR active phonon mode of the MWNTs, as shown in Figure 3(a). The characteristic stretching modes of primary amine groups (appeared at 


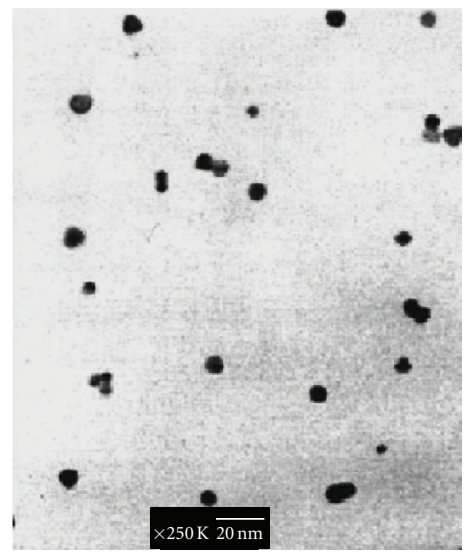

(a)

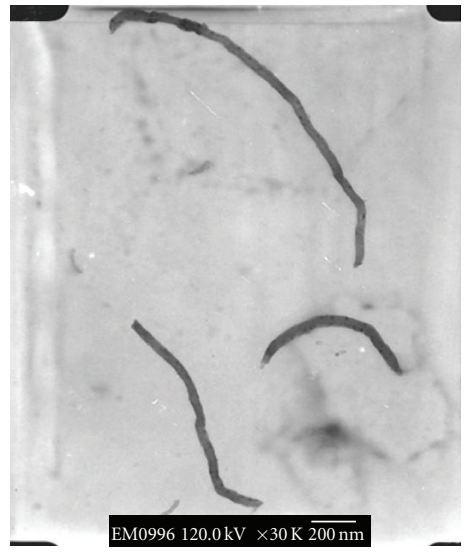

(b)

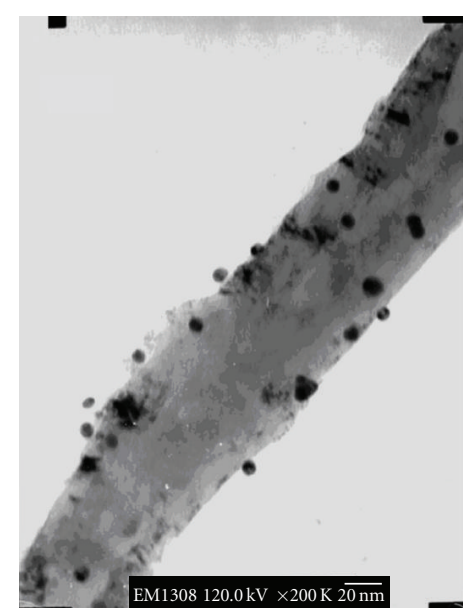

(c)

Figure 2: TEM for (a) Au-NPs (b) MWNT-SH (c) Au-MWNT-SH complex.

3298, 3379 and $1600 \mathrm{~cm}^{-1}$ ) and thiol groups (appeared at $2553 \mathrm{~cm}^{-1}$ ) belong to orthomercaptoaniline were revealed in Figure 3(b). The plausible explanation for the stretching modes of MWNT-SH appeared at around $3264 \mathrm{~cm}^{-1}$ with broad range is that the lone pair of nitrogen atom of orthomercaptoaniline molecules spreads over the electronic charge onto the surface of MWNT through the formation of $\mathrm{p} \pi-\mathrm{p} \pi$ weak interaction between the primary amine groups of orthomercaptoaniline molecules and the surface of MWNTs. The representative peak of thiol group at $2553 \mathrm{~cm}^{-1}$ is also found in the MWNT-SH. These results implied the formation of terminated thiol groups at the ends and on the sidewalls of the MWNTs (MWNT-SH), as shown in Figure 3(c). Furthermore, the characteristic stretching modes of thiol groups with broad range had vanished. It indicates that the gold nanoparticles are assembled to nanotubes via noncovalent interaction between $-\mathrm{SH}$ groups of thiol-terminated MWNTs and gold nanoparticles to form Au-MWNT-SH complex by taking advantage of surface active and strong sulfur affinity of Au-NPs, as shown in Figure 3(d).

3.4. UV-Vis Spectra. The electronic spectra of the solution of compounds, namely, MWNTs, MWNT-SH, and AuMWNT-SH complex are performed in polar DMAc and as well as nonpolar chloroform solvents but Au-NPs are performed in water, because in other solvent it aggregates, as shown in Figure 4. The sharp absorption peak appeared at $335 \mathrm{~nm}$ is assigned as the $\pi-\pi^{*}$ transition of benzene ring [42] (see, Figure 4(b)). Again these peaks are appeared at $299 \mathrm{~nm}\left(\pi-\pi^{*}\right)$ and $242 \mathrm{~nm}\left(n-\sigma^{*}\right)$ in chloroform solvent respectively. There is no difference in peaks between the refluxed orthomercaptoaniline and orthomercaptoaniline, but the characteristic peak (326 nm in DMAc and $282 \mathrm{~nm}$ in chloroform) of the MWNTs is red-shifted due to the transition of $\pi$ electron of MWNTs to $\pi^{*}$ orbital in benzene ring of orthomercaptoaniline. This is the strong evidence in

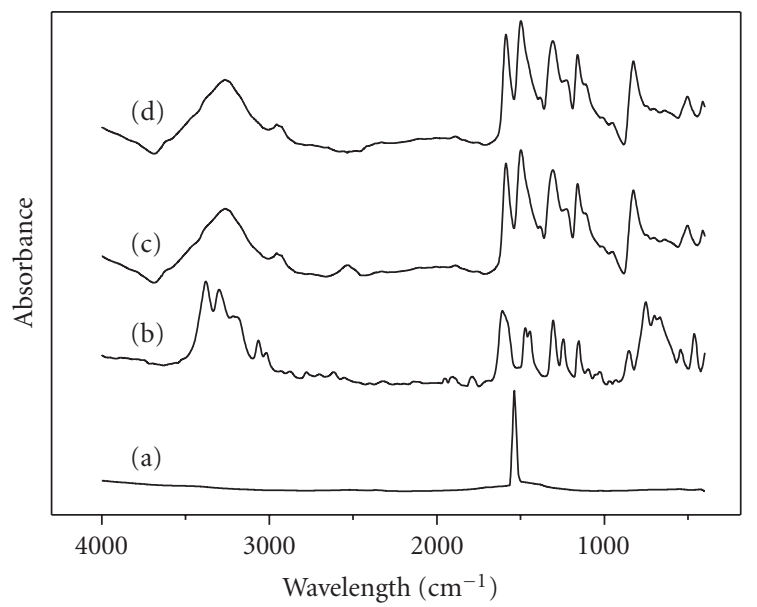

FIGURE 3: FTIR spectra of (a) MWNT, (b) orthomercaptoaniline, (c) MWNT-SH, (d) Au-MWNT-SH complex.

favor of the noncovalent attachment of orthomercaptoaniline onto the surface of MWNTs [15, 30]. The characteristic peak of the Au-NPs in water is appeared at $515 \mathrm{~nm}$, caused by surface plasmon resonance as shown in Figure 4(c). All these peaks (i.e., 331 and $515 \mathrm{~nm}$ in polar DMAc) in AuMWNT-SH complex become broad and peak of MWNT-SH corresponding to $336 \mathrm{~nm}$ is slightly blue shifted to $331 \mathrm{~nm}$ whereas Au-NPs iare independent of wave-length means of red shift compared to the pristine sample (Figure $4(\mathrm{~d})$ ), but, $299 \mathrm{~nm}$ peak of MWNT-SH in nonpolar chloroform shifted to $334 \mathrm{~nm}$ (red shift) and $515 \mathrm{~nm}$ peak of Au-NPs also shifted to $334 \mathrm{~nm}$ (blue shift) in Au-MWNT-SH complex. Thus the existence of the feature of peaks of orthomercaptoaniline strongly suggest the binding of orthomercaptoaniline on the surface of MWNTs, most presumably through $\pi-\pi$ interactions between the benzene ring units of orthomercaptoaniline and the sidewall of MWNTs [43]. The binding of Au-NPs through the thiol end of orthomercaptoaniline 


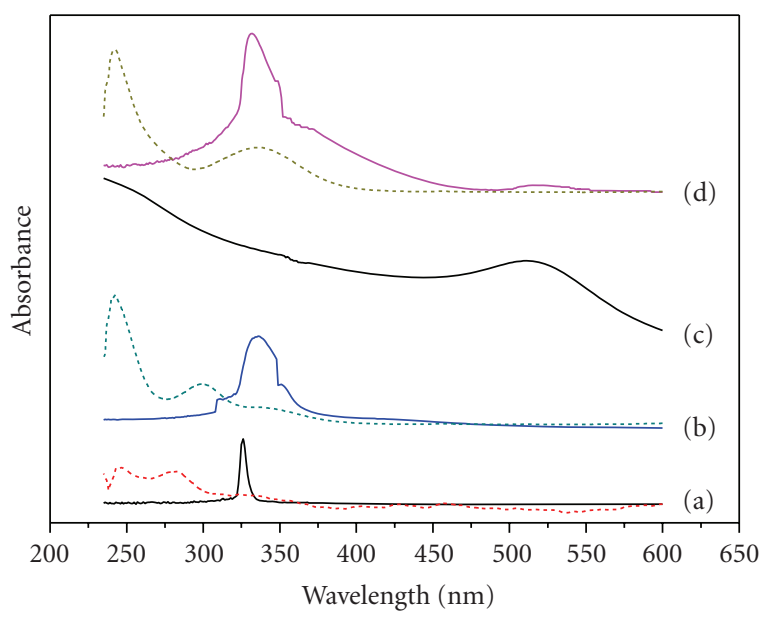

Figure 4: UV-vis absorption spectra of (a) orthomercaptoaniline, (b) MWNT-SH, (c) Au-Nps, (d) Au-MWNT-SH complex, and the solid lines are samples in DMAc (polar) solvent and the dash lines are in $\mathrm{CHCl}_{3}$ (nonpolar) solvent except (c) Au-Nps is in $\mathrm{H}_{2} \mathrm{O}$.

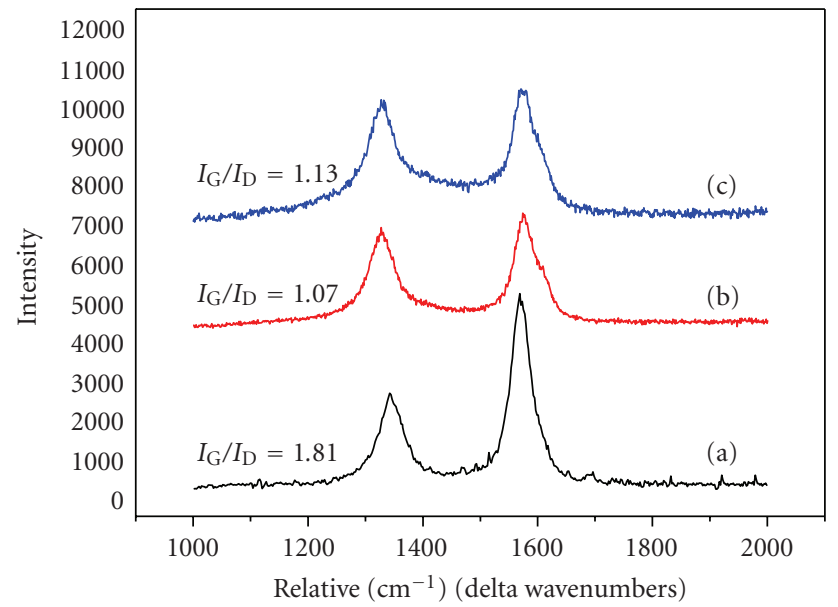

Figure 5: Raman spectra of (a) MWNT (b) MWNT-SH (c) AuMWNT-SH complex.

can be clearly seen from the obvious typical surface plasmon band of Au-NPs centered at $515 \mathrm{~nm}$ (in DMAc) and $334 \mathrm{~nm}$ (in chloroform) with broaden peak. Thus we may conclude that this result is in good agreement with successful assembly of the Au nanoparticles on MWNTs by thiol-functionalized MWNT procedure in our experiments. The spectrum of Au-MWNT-SH complex is different from polar to nonpolar solvent. The metal to ligand charge transfer (MLCT) spectra of Au-MWNT-SH complex are observed when Au is in low oxidation state (zero oxidation) and ligand has low-lying acceptor orbital, for example, $\pi^{*}$ orbital of benzene ring of orthomercaptoaniline. It is sensitive to solvent polarities and observed in UV region. So, we may conclude that the AuMWNT-SH have solvatochromism effect.

3.5. Raman Spectra. The Raman spectra of MWNTs, MWNT-SH and Au-MWNT-SH complexes are shown in

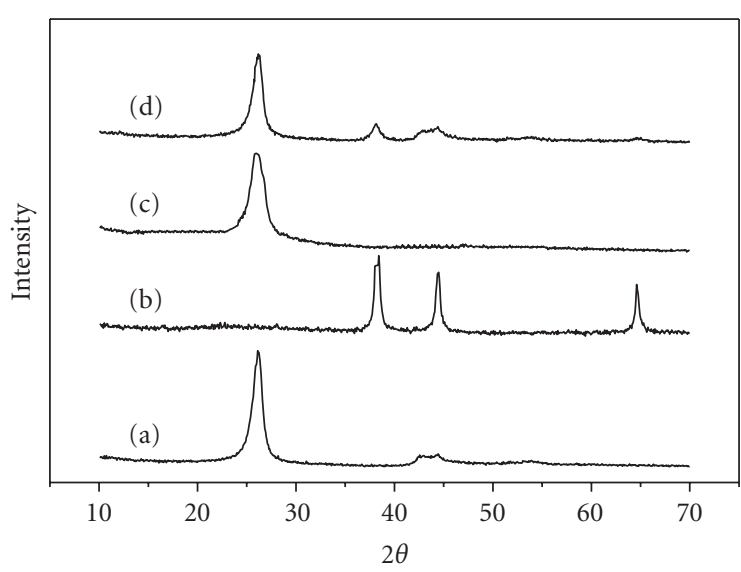

Figure 6: XRD patterns of (a) MWNTs (b) Au-NPs (c) MWNT-SH (d) Au-MWNT-SH complex.

Figure 5. Upon deposition of Au-NPs onto the thiol group of MWNT-SH, both Raman spectra patterns and relative intensities of $\mathrm{G}$ and $\mathrm{D}$ band have been changed slightly. Firstorder Raman spectroscopy of MWNTs shows that a strong band appeared at $1580 \mathrm{~cm}^{-1}$ (G mode) is the Raman-allowed phonon high-frequency mode, and a disordered-induced peak appeared at $1355 \mathrm{~cm}^{-1}$ (D mode) may originate from the defects in the curved grapheme sheets and tube ends. The $G$ and D modes turn to red shift in Figures 5(b) and 5(c). The probable explanation for this behavior was that the average $\mathrm{C}-\mathrm{C}$ bond in MWNTs was strengthened because of the additional electron density on MWNTs, and G mode red shifted by $\pi-\pi$ stacking interaction. Comparing the $I_{\mathrm{G}} / I_{\mathrm{D}}$ ratios of the samples, which are 1.81 for MWNTs, 1.07 for MWNT-SH, and 1.13 for Au-MWNT-SH complexes reveals that with increasing the denominator D-band, $I_{\mathrm{G}} / I_{\mathrm{D}}$ ratio decreases due to the attachment of both orthomercaptoaniline onto the surface of the MWNTs and attachment of Au-NPs onto the thiol group of MWNT-SH, respectively. The thiol-activated and Au-NPs treatment indeed increases the disordering onto the surface of MWNTs. This finding indicates the presence of a few defects at both the ends and the sidewalls of the MWNT-SH. The Raman spectrum of the Au-MWNT-SH complex is examined and is shown in Figure 5(c).

3.6. WAXD Measurement. The crystallization of Au-NPs on the surface of nanotubes was investigated by WAXD. Unlike TEM, the WAXD pattern of a sample can probe a large number of crystallites that are statistically oriented. Diffraction patterns of Au-NPs (Figure 6(b)) appeared at about $38.2^{\circ}, 44.4^{\circ}, 64.8^{\circ}$, which correspond to the (111), (200), and (220) planes of the cubic phase of $\mathrm{Au}$, respectively [44]. Figure 6(d) shows the typical wide-angle Xray diffraction patterns of the $\mathrm{Au}$ nanocrystallites of the sample with Au/MWNT-SH weight ratio of $1: 1$. In the Au-MWNT-SH case, the WAXD pattern essentially shows (111) and (200), as shown in Figure 6(d), while the (220) peak is not discernable above the noise in the diffraction pattern. This suggests that orthomercaptoaniline reacted 
with the Au colloids mainly through the (111) and (200) planes. As a result, the (111) and (200) planes of the Au colloids lie mainly in parallel to the nanotube surface. This phenomenon is probably due to the deposition of a layer of $\mathrm{Au}-\mathrm{NPs}$ to the smooth surface of nanotube leading to parallel orientation of gold (111) and (200) face along the nanotube surface.

\section{Conclusions}

Gold nanoparticle complexes with CNTs using orthomercaptoaniline as a molecular coupler were produced. The process allowed the easy organization of Au-NPs onto the surface of the MWNTs. The noncovalent attachment is occurred between the benzene groups of orthomercaptoaniline onto the surface of MWNTs with an ethelenic double bond via electron transfer mechanism. This noncovalent attachment occurs due to the $\pi-\pi$ interaction between $\pi$ electrons of the benzene ring of orthomercaptoaniline and the ethelenic double bond on the surface of the carbon nanotubes. Another noncovalent attachment is occurred between thiol groups of orthomercaptoaniline of MWNT-SH and the AuNPs by the advantage of surface active properties and sulfur affinity property of Au-NPs. This complex formation is supported by TEM, ATR-FTIR, solution spectra, Raman spectra, and X-ray diffraction studies. The IR stretching modes of thiol groups with broad range indicate that the gold nanoparticles are assembled with nanotubes via noncovalent interaction between $-\mathrm{SH}$ groups of thiolterminated MWNTs and gold nanoparticles to form AuMWNT-SH complex by taking advantage of surface active and strong sulfur affinity of Au-NPs. The Au-MWNT-SH complex shows MLCT spectra due to the electron transfer between the zero oxidation state of $\mathrm{Au}$ and $\pi^{*}$ orbital of benzene ring of orthomercaptoaniline and also have solvent polarity effect. Comparing the $I_{\mathrm{G}} / I_{\mathrm{D}}$ ratios of the samples, which are 1.81, 1.07, and 1.13 for MWNTs, MWNT-SH, and Au-MWNT-SH complexes, respectively reveal that with increasing the denominator D-band, $I_{\mathrm{G}} / I_{\mathrm{D}}$ ratio decreases due to the attachment of both orthomercaptoaniline onto the surface of the MWNTs and Au-NPs onto the thiol group of MWNT-SH, respectively. The Au-MWNT-SH complex is formed due to the deposition of a layer of Au-NPs to the smooth surface of nanotube leading to parallel orientation of gold (111) and (200) face along the nanotube surface. The interaction between the gold nanoparticles and nanotubes is quite strong, because washing and centrifugation do not affect them. It is also the worth of mentioning that the Au-MWNT-SH assembles are formed very stable.

\section{Acknowledgments}

The partial financial support of this research by the NSC 92-2113-M-033-004 and Center-of-Excellence Program on Membrane Technology, The ministry of Education, is gratefully acknowledged.

\section{References}

[1] K. Keren, R. S. Berman, E. Buchstab, U. Sivan, and E. Braun, "DNA-templated carbon nanotube field-effect transistor," Science, vol. 302, no. 5649, pp. 1380-1382, 2003.

[2] H. Yan, S. H. Park, G. Finkelstein, J. H. Reif, and T. H. LaBean, "DNA-templated self-assembly of protein arrays and highly conductive nanowires," Science, vol. 301, no. 5641, pp. 18821884, 2003.

[3] M. Zheng, A. Jagota, M. S. Strano, et al., "Structure-based carbon nanotube sorting by sequence-dependent DNA assembly," Science, vol. 302, no. 5650, pp. 1545-1548, 2003.

[4] D. J. Pochan, Z. Chen, H. Cui, K. Hales, K. Qi, and K. L. Wooley, "Toroidal triblock copolymer assemblies," Science, vol. 306, no. 5693, pp. 94-97, 2004.

[5] H. Ago, K. Petritsch, M. S. P. Shaffer, A. H. Windle, and R. H. Friend, "Composites of carbon nanotubes and conjugated polymers for photovoltaic devices," Advanced Materials, vol. 11, no. 15, pp. 1281-1285, 1999.

[6] S. Iijima and T. Ichihashi, "Single-shell carbon nanotubes of 1nm diameter," Nature, vol. 363, no. 6430, pp. 603-605, 1993.

[7] D. J. Styers-Barnett, S. P. Ellison, C. Park, K. E. Wise, and J. M. Papanikolas, "Ultrafast dynamics of single-walled carbon nanotubes dispersed in polymer films," Journal of Physical Chemistry A, vol. 109, no. 2, pp. 289-292, 2005.

[8] S. Auvray, V. Derycke, M. Goffman, A. Filoramo, O. Jost, and J.-P. Bourgoin, "Chemical optimization of self-assembled carbon nanotube transistors," Nano Letters, vol. 5, no. 3, pp. 451-455, 2005.

[9] P. He and M. Bayachou, "Layer-by-layer fabrication and characterization of DNA-wrapped single-walled carbon nanotube particles," Langmuir, vol. 21, no. 13, pp. 6086-6092, 2005.

[10] G. Lu, P. Maragakis, and E. Kaxiras, "Carbon nanotube interaction with DNA," Nano Letters, vol. 5, no. 5, pp. 897900, 2005.

[11] S. Li, P. He, J. Dong, Z. Guo, and L. Dai, "DNA-directed self-assembling of carbon nanotubes," Journal of the American Chemical Society, vol. 127, no. 1, pp. 14-15, 2005.

[12] J. Du, L. Fu, Z. Liu, et al., "Facile route to synthesize multiwalled carbon nanotube/zinc sulfide heterostructures: optical and electrical properties," Journal of Physical Chemistry $B$, vol. 109, no. 26, pp. 12772-12776, 2005.

[13] C. J. Kiely, J. Fink, M. Brust, D. Bethell, and D. J. Schiffrin, "Spontaneous ordering of bimodal ensembles of nanoscopic gold clusters," Nature, vol. 396, no. 6710, pp. 444-446, 1998.

[14] K. M. Mayya, N. Jain, A. Gole, D. Langevin, and M. Sastry, "Time-dependent complexation of glucose-reduced gold nanoparticles with octadecylamine Langmuir monolayers," Journal of Colloid and Interface Science, vol. 270, no. 1, pp. 133139, 2004.

[15] L. Liu, T. Wang, J. Li, et al., "Self-assembly of gold nanoparticles to carbon nanotubes using a thiol-terminated pyrene as interlinker," Chemical Physics Letters, vol. 367, no. 5-6, pp. 747-752, 2003.

[16] J. Shi, Z. Wang, and H.-L. Li, "Selfassembly of gold nanoparticles onto the surface of multiwall carbon nanotubes functionalized with mercaptobenzene moieties," Journal of Nanoparticle Research, vol. 8, no. 5, pp. 743-747, 2006.

[17] J.-H. Kim and T. R. Lee, "Hydrogel-templated growth of large gold nanoparticles: synthesis of thermally responsive hydrogel-nanoparticle composites," Langmuir, vol. 23, no. 12, pp. 6504-6509, 2007. 
[18] M. Lahav, E. A. Weiss, Q. Xu, and G. M. Whitesides, "Coreshell and segmented polymer-metal composite nanostructures," Nano Letters, vol. 6, no. 9, pp. 2166-2171, 2006.

[19] N. Kocharova, T. Ääritalo, J. Leiro, J. Kankare, and J. Lukkari, "Aqueous dispersion, surface thiolation, and direct selfassembly of carbon nanotubes on gold," Langmuir, vol. 23, no. 6, pp. 3363-3371, 2007.

[20] R. H. Terrill, T. A. Postlethwaite, C.-H. Chen, et al., "Monolayers in three dimensions: NMR, SAXS, thermal, and electron hopping studies of alkanethiol stabilized gold clusters," Journal of the American Chemical Society, vol. 117, no. 50, pp. 1253712548, 1995.

[21] M. Haruta and M. Daté, "Advances in the catalysis of Au nanoparticles," Applied Catalysis A, vol. 222, no. 1-2, pp. 427 437, 2001.

[22] H. W. Ch. Postma, T. Teepen, Z. Yao, M. Grifoni, and C. Dekker, "Carbon nanotube single-electron transistors at room temperature," Science, vol. 293, no. 5527, pp. 76-79, 2001.

[23] B. M. Quinn, C. Dekker, and S. G. Lemay, "Electrodeposition of noble metal nanoparticles on carbon nanotubes," Journal of the American Chemical Society, vol. 127, no. 17, pp. 6146-6147, 2005.

[24] D. A. Stewart and F. Léonard, "Energy conversion efficiency in nanotube optoelectronics," Nano Letters, vol. 5, no. 2, pp. 219-222, 2005.

[25] D. Baskaran, J. W. Mays, X. P. Zhang, and M. S. Bratcher, "Carbon nanotubes with covalently linked porphyrin antennae: photoinduced electron transfer," Journal of the American Chemical Society, vol. 127, no. 19, pp. 6916-6917, 2005.

[26] V. Derycke, R. Martel, J. Appenzeller, and Ph. Avouris, "Carbon nanotube inter- and intramolecular logic gates," Nano Letters, vol. 1, no. 9, pp. 453-456, 2001.

[27] P. M. Ajayan, O. Stephan, Ph. Redlich, and C. Colliex, "Carbon nanotubes as removable templates for metal oxide nanocomposites and nanostructures," Nature, vol. 375, no. 6532, pp. 564-567, 1995.

[28] G. Che, B. B. Lakshmi, C. R. Martin, and E. R. Fisher, "Metalnanocluster-filled carbon nanotubes: catalytic properties and possible applications in electrochemical energy storage and production," Langmuir, vol. 15, no. 3, pp. 750-758, 1999.

[29] R. J. Chen, Y. Zhang, D. Wang, and H. Dai, "Noncovalent sidewall functionalization of single-walled carbon nanotubes for protein immobilization," Journal of the American Chemical Society, vol. 123, no. 16, pp. 3838-3839, 2001.

[30] Z. Yang, X. Chen, C. Chen, et al., "Noncovalent-wrapped sidewall functionalization of multiwalled carbon nanotubes with polyimide," Polymer Composites, vol. 28, no. 1, pp. 36-41, 2007.

[31] Z. Yang, X. Chen, Y. Pu, et al., "Facile approach to obtain individual-nanotube dispersion at high loading in carbon nanotubes/polyimide composites," Polymers for Advanced Technologies, vol. 18, no. 6, pp. 458-462, 2007.

[32] P. Petrov, F. Stassin, C. Pagnoulle, and R. Jérôme, "Noncovalent functionalization of multi-walled carbon nanotubes by pyrene containing polymers," Chemical Communications, no. 23, pp. 2904-2905, 2003.

[33] Z. Zhang, J. Zhang, P. Chen, B. Zhang, J. He, and G.-H. $\mathrm{Hu}$, "Enhanced interactions between multi-walled carbon nanotubes and polystyrene induced by melt mixing," Carbon, vol. 44, no. 4, pp. 692-698, 2006.

[34] X. Lou, R. Daussin, S. Cuenot, et al., "Synthesis of pyrenecontaining polymers and noncovalent sidewall functionalization of multiwalled carbon nanotubes," Chemistry of Materials, vol. 16, no. 21, pp. 4005-4011, 2004.
[35] J. S. Kavakka, S. Heikkinen, I. Kilpeläinen, M. Mattila, H. Lipsanen, and J. Helaja, "Noncovalent attachment of pyropheophorbide a to a carbon nanotube," Chemical Communications, no. 5, pp. 519-521, 2007.

[36] D. A. Britz and A. N. Khlobystov, "Noncovalent interactions of molecules with single walled carbon nanotubes," Chemical Society Reviews, vol. 35, no. 7, pp. 637-659, 2006.

[37] R. Zanella, E. V. Basiuk, P. Santiago, et al., "Deposition of gold nanoparticles onto thiol-functionalized multiwalled carbon nanotubes," Journal of Physical Chemistry B, vol. 109, no. 34, pp. 16290-16295, 2005.

[38] B. Pan, D. Cui, F. Gao, and R. He, "Attachment of gold nanoparticles on multi-walled carbon nanotubes," Nanoscience, vol. 11, no. 2, pp. 95-101, 2006.

[39] P. W. Chiu, G. S. Duesberg, U. Dettlaff-Weglikowska, and S. Roth, "Interconnection of carbon nanotubes by chemical functionalization," Applied Physics Letters, vol. 80, no. 20, pp. 3811-3813, 2002.

[40] D. M. Delozier, D. M. Tigelaar, K. A. Watson, et al., "Investigation of ionomers as dispersants for single wall carbon nanotubes," Polymer, vol. 46, no. 8, pp. 2506-2521, 2005.

[41] M. F. Islam, E. Rojas, D. M. Bergey, A. T. Johnson, and A. G. Yodh, "High weight fraction surfactant solubilization of single-wall carbon nanotubes in water," Nano Letters, vol. 3, no. 2, pp. 269-273, 2003.

[42] Y. Geng, J. Li, X. Jing, and F. Wang, "Interaction of Nmethylpyrrolidone with doped polyaniline," Synthetic Metals, vol. 84, no. 1-3, pp. 97-98, 1997.

[43] R. J. Chen, Y. Zhang, D. Wang, and H. Dai, "Noncovalent sidewall functionalization of single-walled carbon nanotubes for protein immobilization," Journal of the American Chemical Society, vol. 123, no. 16, pp. 3838-3839, 2001.

[44] X. Feng, C. Mao, G. Yang, W. Hou, and J.-J. Zhu, "Polyaniline/Au composite hollow spheres: synthesis, characterization, and application to the detection of dopamine," Langmuir, vol. 22, no. 9, pp. 4384-4389, 2006. 

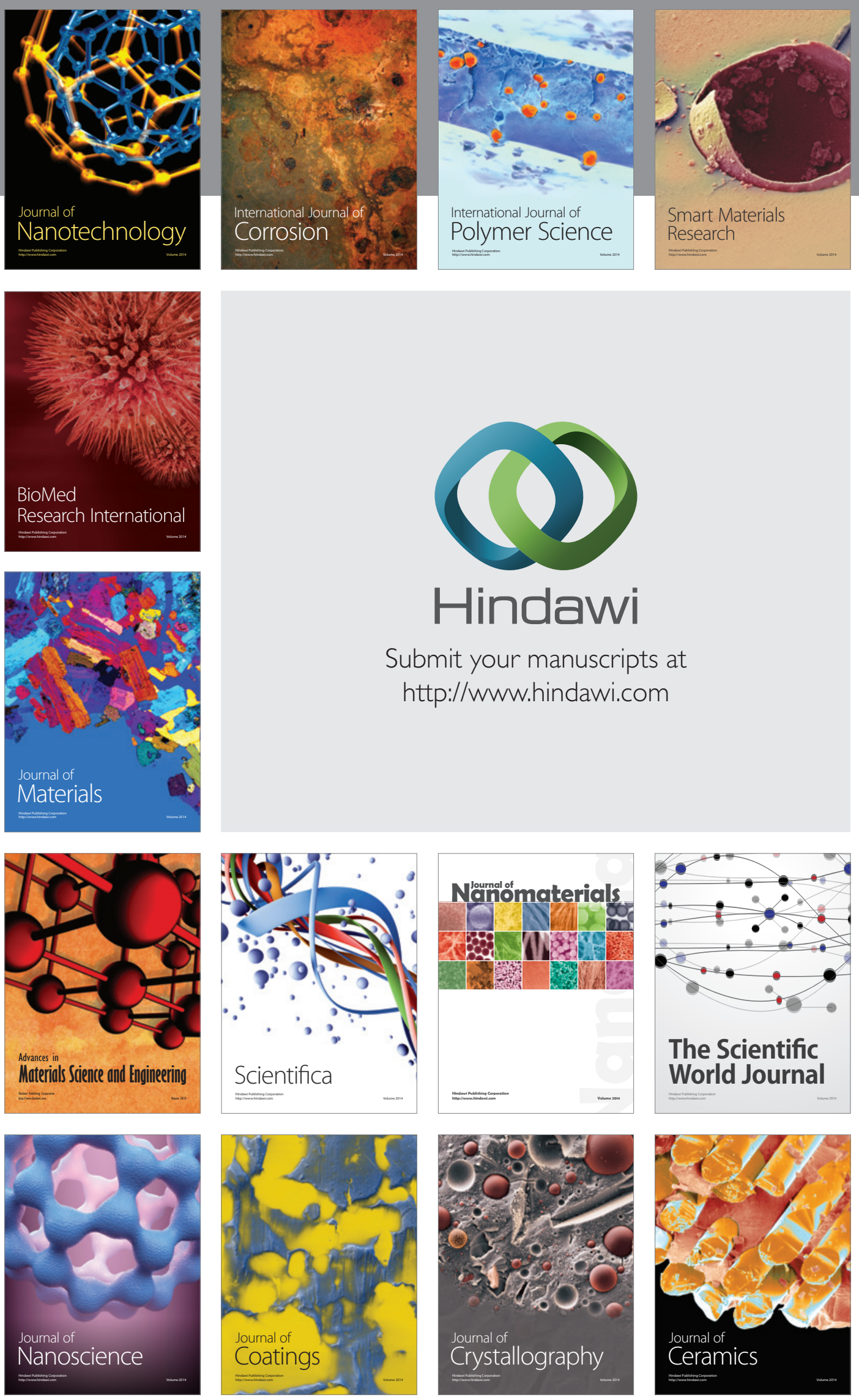

The Scientific World Journal

Submit your manuscripts at

http://www.hindawi.com

\section{World Journal}

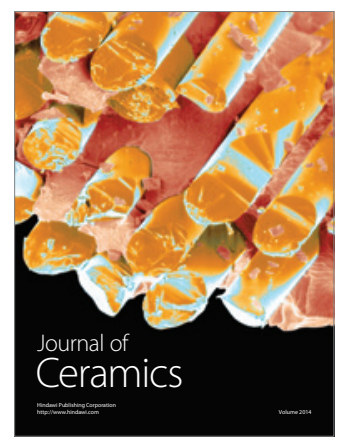

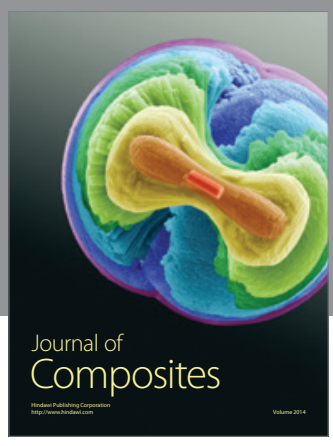
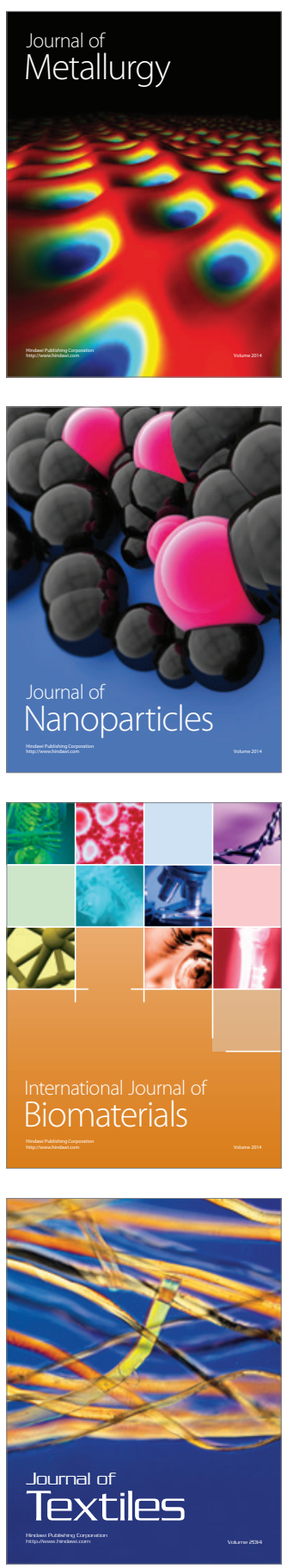